\title{
Preoperative Embolization of Cerebellar Hemangioblastoma with Onyx: Report of Three Cases
}

\author{
Gi Won Shin, MD', Hae Woong Jeong, MD', Jeong Hwa Seo, MD², Sung Tae Kim, MD², \\ Hye Jung Choo, MD', Sun Joo Lee, MD'
}

Hemangioblastoma is a benign and highly vascular tumor. Complete surgical resection of highly vascular tumor such as hemangioblastoma may be challenging due to excessive bleeding. Preoperative embolization of these lesions may decrease the intraoperative blood loss and facilitate excision. We report three cases of cerebellar hemangioblastomas that were embolized using Onyx.

Key Words : Hemangioblastoma; Endovascular procedure; Embolization/therapy; Onyx

Hemangioblastoma is a benign and highly vascular tumor [1]. The treatment of choice for tumors containing mural nodules such as hemangioblastoma is total resection. However, as this lesion usually originated in the posterior cranial fossa is highly vascular tumor, excessive arterial bleeding occasionally occurs in the narrow operative field. In these cases, it is hard to perform total resection. If successful preoperative embolization of hemangioblastoma is performed, facilitate and total resection can be done [2]. We presented experiences with ethylene vinylalcohol copolymer, Onyx (ev3 Inc., Irvine, California, USA) embolization of hemangioblastoma before surgical resection.

\footnotetext{
'Departments of Diagnostic Radiology, ${ }^{2}$ Neurology, and ${ }^{3}$ Neurosurgery, Busan Paik Hospital, Inje University, Busan, Korea Received August 14, 2013; accepted after revision November 14, 2013.

Correspondence to: Hae Woong Jeong, MD, Department of Diagnostic Radiology, Busan Paik Hospital, Inje University College of Medicine, 75 Bokji-ro, Busanjin-gu, Busan 614-735, Korea.

Tel. 82.51.890.6547 Fax. 82.51.896.1085

E-mail: hwjeong2000@lycos.co.kr

This is an Open Access article distributed under the terms of the Creative Commons Attribution Non-Commercial License (http://creativecommons.org/licenses/by-nc/3.0) which permits unrestricted non-commercial use, distribution, and reproduction in any medium, provided the original work is properly cited.
}

\section{CASE REPORTS}

\section{Case 1}

A 43-year-old man presented with a three day history of progressive headache. Brain MRI revealed a $2.7 \times$ $2.5 \mathrm{~cm}$ sized posterior fossa tumor showing significant enhancement with multiple signal void vascular structures within the tumor (Fig. 1A). The differential diagnosis included hemangioblastoma, metastasis and high grade glioma. A diagnostic vertebral angiogram demonstrated a significant vascular blush supplied mostly from the right superior cerebellar artery. There was obvious arteriovenous (AV) shunt showing a highly vascular lesion, consistent with a hemangioblastoma (Fig. 1B). The patient underwent embolization of core feeder vessel originated off the right superior cerebellar artery using Onyx, resulting near complete resolution of the tumor blush (Fig. 1C, D). Near total resection of the tumor was performed without excessive bleeding or other complication. Post-operative MRI revealed volume reduction over $90 \%$ with nearly total regression of the tortuous flow voids. The tumor was pathologically confirmed as hemangioblastoma. Histological examination of the excised tumor revealed that the hemangioblastoma was composed of stromal cells, 


\section{Gi Won Shin, et al.}
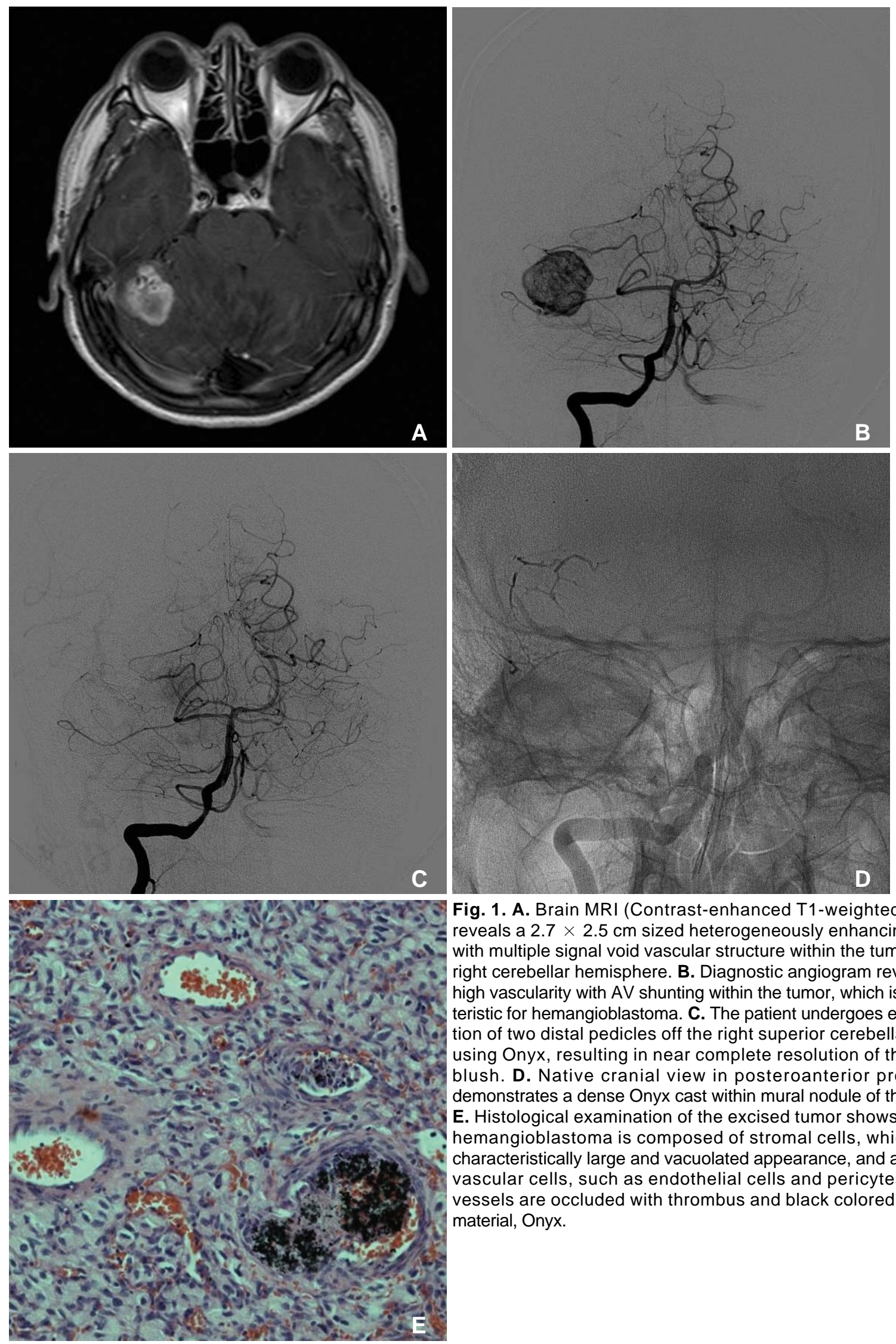

Fig. 1. A. Brain MRI (Contrast-enhanced T1-weighted image) reveals a $2.7 \times 2.5 \mathrm{~cm}$ sized heterogeneously enhancing tumor with multiple signal void vascular structure within the tumor in the right cerebellar hemisphere. B. Diagnostic angiogram reveals the high vascularity with $A V$ shunting within the tumor, which is characteristic for hemangioblastoma. C. The patient undergoes embolization of two distal pedicles off the right superior cerebellar artery using Onyx, resulting in near complete resolution of the tumor blush. D. Native cranial view in posteroanterior projection demonstrates a dense Onyx cast within mural nodule of the tumor. E. Histological examination of the excised tumor shows that the hemangioblastoma is composed of stromal cells, which show characteristically large and vacuolated appearance, and abundant vascular cells, such as endothelial cells and pericytes. Nidus vessels are occluded with thrombus and black colored embolic material, Onyx. 


\section{Embolization of Cerebellar Hemangioblastoma}

which showed characteristically large and vacuolated appearance, and abundant vascular cells, such as endothelial cells and pericytes. Nidus vessels were occluded with thrombus and black colored embolic material, Onyx (Fig. 1E). After surgical resection, the patient presented gradual regression of the headache.

\section{Case 2}

A 53-year-old man presented with a 15 day history of progressive vertigo and occipital headache. Brain MRI revealed a $3.2 \times 2.2 \mathrm{~cm}$ cystic tumor with a $1.0 \times 0.5$ $\mathrm{cm}$ sized densely enhancing mural nodule in the right cerebellum (Fig. 2A). A diagnostic vertebral angiogram revealed early intense tumor staining in the right cerebellum from the right superior cerebellar artery with AV shunt showing a highly vascular lesion (Fig. 2B). The patient underwent Onyx embolization of core feeder vessel originated off the right superior cerebellar artery and tumor blush was complete resolved (Fig. 2C). Near total resection of the tumor was performed with minimal blood loss. Post operative MRI revealed volume reduction over $90 \%$ of enhancing mural nodule with nearly total regression of the cystic portion. The tumor was pathologically confirmed as hemangioblastoma. After surgical resection, vertigo and occipital headache were gradually resolved.

\section{Case 3}

An 18-year-old man presented with a month history of progressive headache. Brain MRI revealed a $5.3 \times$ $3.8 \mathrm{~cm}$ sized large cystic tumor with a $2.0 \times 1.6$ $\mathrm{cm}$ sized heterogeneously enhancing mural nodule in the right cerebellum with tumor effect. Tumor effect was causing effacement and displacement of the fourth
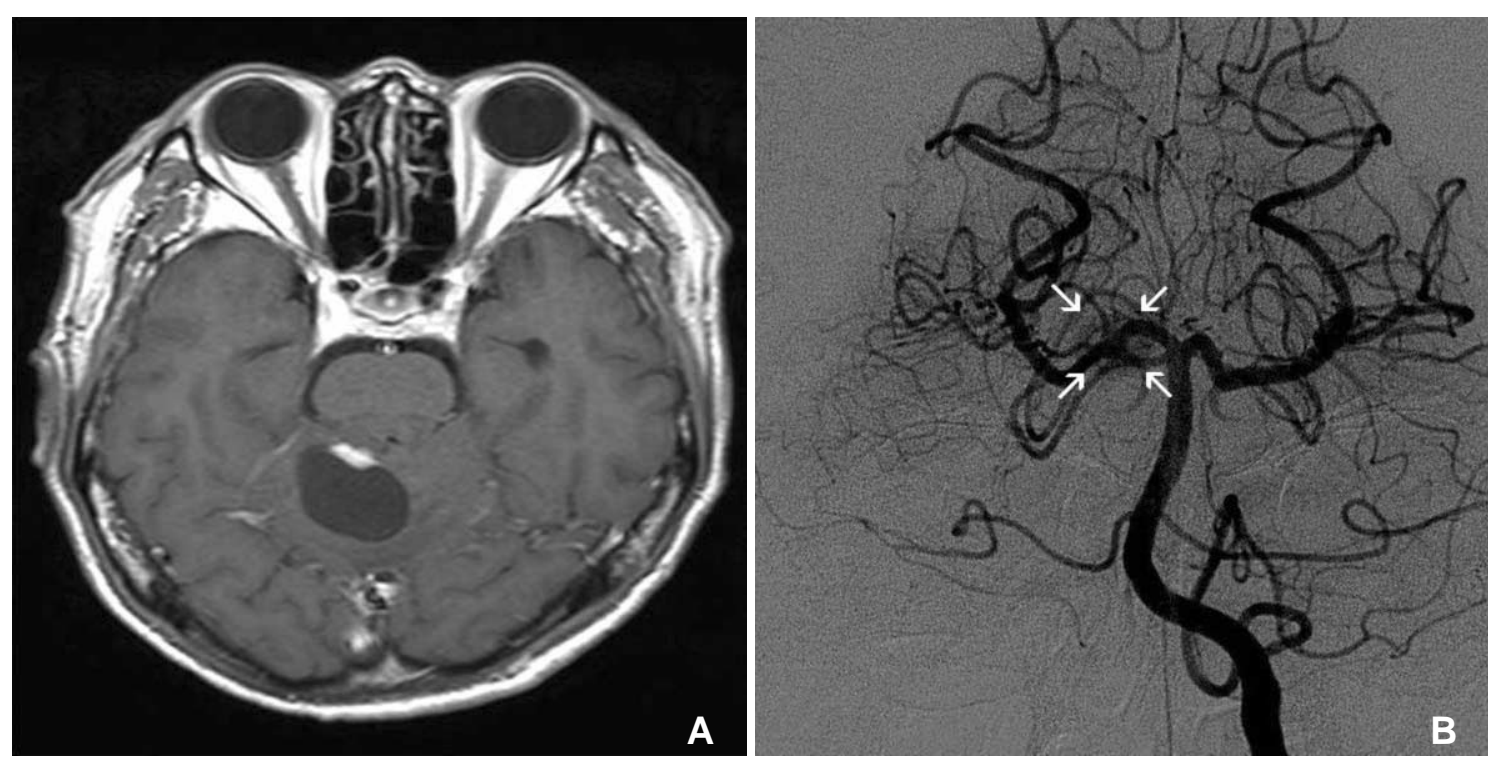

Fig. 2. A. Brain MRI (Contrast-enhanced T1-weighted image) reveals a cystic tumor with a densely enhancing mural nodule in the right cerebellum. B. A diagnostic vertebral angiogram reveals early intense tumor staining (arrows) in the right cerebellum from the right superior cerebellar artery. C. The patient undergoes embolization of core feeder vessel originated off the right superior cerebellar artery using Onyx. Final angiogram shows near complete resolution of the tumor blush. 

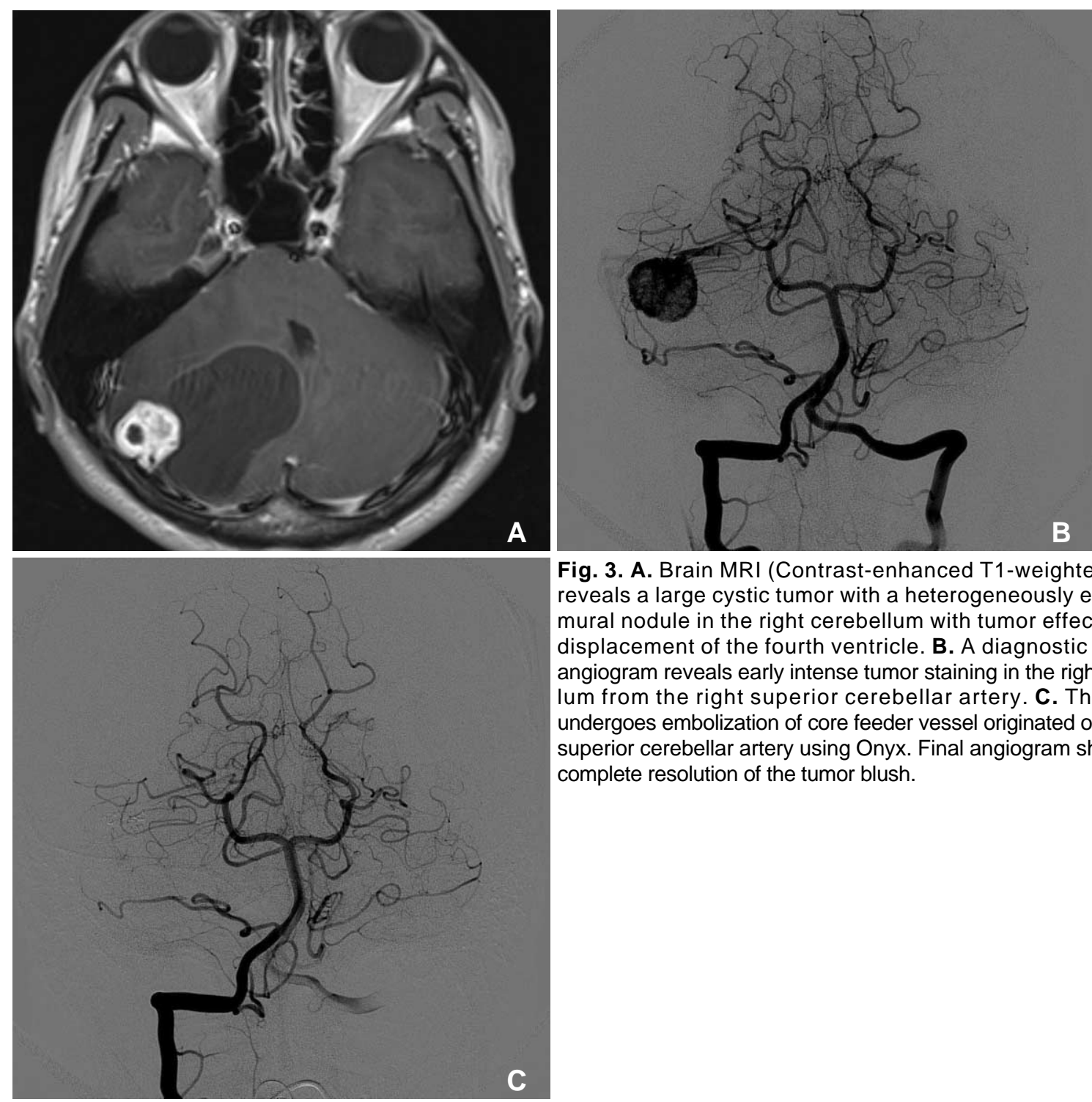

Fig. 3. A. Brain MRI (Contrast-enhanced T1-weighted image) reveals a large cystic tumor with a heterogeneously enhancing mural nodule in the right cerebellum with tumor effect causing displacement of the fourth ventricle. B. A diagnostic vertebral angiogram reveals early intense tumor staining in the right cerebellum from the right superior cerebellar artery. C. The patient undergoes embolization of core feeder vessel originated off the right superior cerebellar artery using Onyx. Final angiogram shows near complete resolution of the tumor blush.

ventricle (Fig. 3A). A diagnostic vertebral angiogram revealed early intense tumor staining in the right cerebellum from the right superior cerebellar artery with AV shunt showing a highly vascular lesion (Fig. 3B). The patient underwent embolization of a pedicle off the hemispheric branch of the right superior cerebellar artery using Onyx, resulting in near complete resolution of the tumor blush (Fig. 3C). Near total resection of the tumor was performed with minimal blood loss. Post embolization non-enhanced CT revealed radiopaque embolic agent, Onyx in the mural nodule. Post operative MRI revealed volume reduction over $90 \%$ of enhancing mural nodule with nearly total regression of the cystic portion. The tumor was pathologically confirmed as hemangioblastoma. After surgical resection, the patient presented gradual regres- sion of the headache.

\section{DISCUSSION}

Preoperative embolization of cranial hemangioblastomas is used with the purpose of facilitating surgical resection of these lesions. Several embolic agents have been used for decreasing the arterial supply to the lesion with adequate capillary bed penetration. Embolic materials are divided into three categories: mechanical devices, particles, and liquid agents. Mechanical devices, such as balloons or coils, are able to occlude large feeders but they lack the ability to penetrate the tumor bed. Particles, such as polyvinyl alcohol, Embospheres (Guerbet Biomedical, Louvres, France), and Gelfoam (Upjohn Co., Kalamazoo, MI, USA), 


\section{Embolization of Cerebellar Hemangioblastoma}

have also been used. Despite smaller particles have the advantage of better tumor bed penetration, their use also carries a higher risk of inadvertent non-target embolization [3]. Takeuchi et al [4]. reported their series of hemangioblastomas treated with preoperative embolization using primarily particles and concluded that presurgical embolization is useful to reduce operative complications when $80 \%$ or more of tumor could be embolized. Three patients had temporary neurological complications as a result of the embolization. Eskridge et al [5]. reported that one patient experienced worsening of hydrocephalus after embolization, requiring emergency craniotomy and surgical resection. Cornelius et al [6]. reported different clinical outcome of preoperative particulate embolization between spinal and cerebellar hemangioblastomas. Although the outcome of embolization in spinal hemangioblastoma was favorable, that of all cerebellar hemangioblastomas (three patients) was not; acute tumor bleeding and death. This result was probably due to smaller capillary size of cerebellar hemangioblastoma causing venous congestion and subsequent rupture.

Liquid embolic agents (N-butyl cyanoacrylate (NBCA) and Onyx) have the best combination of durability and penetration, being able to devascularize the tumor reaching its core. The major disadvantage associated with NBCA embolization is that the glue polymerizes very rapidly when it contacts an ionic solution such as blood. It increases the risk of gluing the microcatheter in place. Onyx is a non-adhesive liquid embolic agent (ethylene-vinyl alcohol) dissolved in an organic solvent, dimethyl sulfoxide. With Onyx, fewer arterial feeders require catheterization because of the diffusion properties of the agent. It allows deep penetration of the tumor capillary bed, which often results in artery-to-artery embolization of tumor feeders [7]. Another major advantage of this agent in tumor embolization was that reflux along the infusion catheter could be tolerated without requiring removal of the catheter. Unlike NBCA, Onyx can be injected much more slowly, on the order of minutes. The injection can even be discontinuous, thereby allowing angiographic assessment of the embolization and improving the control of embolic delivery. Another advantage of Onyx is its excellent surgical handling properties. Because the tantalum is black, in the operating room, the embolized vessels are easy to see. Onyx includes its radiopacity, durability, and availability in commercial formulations and the relatively modest inflammatory reaction that it generates.

Onyx embolization also has some disadvantages. Injection over long distances $(>2 \mathrm{~cm})$ in small diameter or low-flow vessels is difficult and it can migrate into the veins [8]. Another consideration with the use of Onyx includes prolonged fluoroscopic times. We noted several cases in which a single session of Onyx embolization approached 1 hour in duration [3].

Given the advantageous features of Onyx, the need for fewer arterial catheterization, excellent tumor penetration, and low complication profile, we believe that its use may actually be effective and safe compared with other agents [1-3].

As shown in these three cases, Onyx could be useful for preoperative obliteration of highly vascular tumor, such as hemangioblastoma. If devascularization of hypervascular tumor was achieved, tumor resection is significantly simplified and hemorrhagic complication can be minimal. As Onyx is favorable to achieve deep penetration into the core feeding artery, it may be better embolic agent than others for successful preoperative embolization of hypervascular tumor.

\section{References}

1. Horvathy DB, Hauch EF, Ogilvy CS, Hopkins LN, Levy EI, Siddiqui AH. Complete preoperative embolization of hemangioblastoma vessels with Onyx 18. J Clin Neurosci 2011;18:401-403

2. Dabus G, Pryor J, Spilberg G, Samaniego EA, Nogueira RG. Embolization of intra-axial hypervascular tumors with Onyx: report of three cases. J Neurointerv Surg 2013;5:177-180

3. Gore P, Theodore N, Brasiliense L, Kim LJ, Garrett M, Nakaji P, et al. The utility of Onyx for preoperative embolization of cranial and spinal tumor. Neurosurgery 2008;62:1204-1212

4. Takeuchi S, Tanaka R, Fujii Y, Abe H, Ito Y. Surgical treatment of hemangioblastomas with presurgical endovascular embolization. Neurol Med Chir (Tokyo) 2001;41:246-251

5. Eskridge JM, McAuliffe W, Harris B, Kim DK, Scott J, Winn HR. Preoperative endovascular embolization of craniospinal hemanigoblastoma. AJNR Am J Neuroradiol 1996;17:525-531

6. Cornelius JF, Saint-Maurice JP, Bresson D, George B, Houdart E. Hemorrhage after particle embolization of hemangioblastomas: comparison of outcomes in spinal and cerebellar lesions. $J$ Neurosurg 2007;106:994-8

7. Seong Eom K, Won Kim D, Sung Choi S, Ha Choi K, Young Kim T. Preoperative embolization of a cerebellar hemangioblastoma using Onyx. Neurol Neurochir Pol 2011;45(3):292-296

8. Gobin YP, Murayama Y, Milanese K, Chow K, Gonzalez NR, Duckwiler GR, et al. Head and neck hypervascular lesions: embolization with ethylene vinyl alcohol copolymer. Radiology 2001;221:309-317 